Erratum

\title{
Field-Theoretical Approach to Static Critical Phenomena in Semi-Infinite Systems
}

\author{
H.W. Diehl and S. Dietrich \\ Sektion Physik der Universität, Theoretische Physik, München, \\ Federal Republic of Germany \\ Z. Phys. B - Condensed Matter 42, 65-86 (1981)
}

In Eq. (IV.33a) on page $77 \varepsilon^{-j}$ should be replaced by $\varepsilon^{-i}$

Equation (IV.62) on page 78 should read

$M_{1}\left(t, h=0, h_{1}=0\right) \sim|t||\ln | t||^{3 /(n+8)}$.

H.W. Diehl

S. Dietrich

Sektion Physik

Universität München

- Theoretische Physik -

Theresienstrasse 37

D-8000 München 2

Federal Republic of Germany 